\title{
Non-isotropic emitter orientation and its implications for efficiency analysis of organic light-emitting diodes
}

\author{
Tobias D. Schmidt*a ${ }^{\mathrm{a}}$, Michael Flämmich ${ }^{\mathrm{b}}$, Bert J. Scholz ${ }^{\mathrm{a}}$, Dirk Michaelis ${ }^{\mathrm{b}}$, Christian Mayr $^{\mathrm{a}}$, \\ Norbert Danz ${ }^{\mathrm{b}}$, Wolfgang Brütting ${ }^{\mathrm{a}}$ \\ a Institute of Physics, University of Augsburg, Universitätsstraße 1, 86135 Augsburg, Germany; \\ ${ }^{\mathrm{b}}$ Fraunhofer Institute for Applied Optics and Precision Engineering, 07745 Jena, Germany
}

Tobias.Schmidt@physik.uni-augsburg.de Wolfgang.Bruetting@physik.uni-augsburg.de

\begin{abstract}
The efficiency of organic light-emitting diodes (OLEDs) is limited since only a small fraction of the consumed electrical power is converted into visible light that is finally extracted to air. Most of the efficiency loss is caused by suboptimal radiative quantum efficiency (RQE) of the emitting guest-host system and by dissipating a huge part of the radiated energy to optical modes such as surface plasmons or waveguided modes, which cannot easily be extracted by common outcoupling structures. In order to increase the external quantum efficiency (EQE) of OLEDs new approaches are needed. Recent studies show that the EQE can be enhanced considerably by horizontally oriented emitters, a feature that is well known for fluorescent emitters and has lately been demonstrated in phosphorescent state-of-the-art OLEDs. By means of optical simulations we investigated the influence of non-isotropic emitter orientation on the effective RQE and the outcoupling factor. We show that in order to achieve a consistent efficiency analysis it is indispensable to account for possible deviations from isotropy. Ignoring these orientation effects leads to significant misinterpretation of the RQE and other factors, which determine the external quantum efficiency of a device. Furthermore, we demonstrate the huge potential for efficiency enhancement of mainly parallel dipole emitter orientation in both fluorescent and phosphorescent OLEDs.
\end{abstract}

Keywords: Organic light-emitting diode, external quantum efficiency, oriented emitting systems, radiative quantum efficiency, outcoupling efficiency 


\section{INTRODUCTION}

In recent years the interest in organic electronics especially in organic light-emitting diodes (OLEDs) has grown rapidly since the first low-voltage OLED has been presented by Tang and van Slyke in 1987. ${ }^{1}$ Since then a huge amount of results has been published in this field of research and the efficiencies of OLEDs could be increased continuously. Therefore, several groups have presented efficiencies for white OLEDs in the range of $60 . .80 \mathrm{~lm} / \mathrm{W}$ and above. ${ }^{2,3}$ These values are close to that of fluorescent tubes and so the entrance of OLEDs into the general lighting sector is predictable, since the small-area display market (e.g. for mobile phones) has already been flooded predominantly by AMOLED (Active Matrix OLED) displays. The advantages of OLEDs are numerous as it is possible to fabricate lightweight, thin and flat large-area devices, which can be transparent, even on flexible substrates. Although the first commercial products for general lighting are already affordable there is still much room for improvement in efficiency and many physical effects are not yet fully understood.

One possible approach to enhance the external quantum efficiency (EQE) of OLEDs is applying oriented phosphorescent emitters that could increase the outcoupling efficiency of a device without any additional costs or complicated extra optical elements. The EQE of an OLED can be calculated using ${ }^{4}$

$$
\mathrm{EQE}=\gamma \cdot \eta_{\mathrm{s} / \mathrm{t}} \cdot \mathrm{q}_{\mathrm{eff}} \cdot \eta_{\mathrm{out}}
$$

Therein $\gamma$ is the so called charge carrier balance which gives the ratio between formed excitons and injected charge carriers. This factor can be close to unity if appropriate blocking layers are used in an OLED. The second factor in this equation, $\eta_{\mathrm{s} / t}$, is the fraction of excitons that are quantum-mechanically allowed to decay under radiation of light with respect to spin selection rules. This factor is $1 / 4$ or 1 for fluorescent and phosphorescent emitters, respectively. ${ }^{5} \mathrm{q}_{\text {eff }}$ is the effective radiative quantum efficiency (RQE) that is determined by the intrinsic quantum efficiency $(q)$ of the emitting system in use and by the cavity effects in an OLED. ${ }^{6} \eta_{\text {out }}$ describes the amount of photons that can leave the system into the surrounding (air) medium. Due to the material and layered stack poperties its value is typically in the range of $15 . .20 \%$ for planar, bottom emitting devices. ${ }^{4,6,7}$ The rest of the generated light is trapped inside the OLED due to total internal reflection and is lost either in substrate or in waveguided modes. ${ }^{8,9}$

Analyzing in more detail, other effects such as coupling to surface plasmon polaritons at the metallic cathode of the device, ${ }^{8-10}$ losses due to a reduced RQE (that can be far away from unity) ${ }^{11}$ and orientation dependent changes of the power dissipation into the different optical channels ${ }^{12,13}$ need to be taken into account. In order to achieve a comprehensive efficiency analysis of state-of-the-art OLEDs all these effects must be considered. Especially emitter orientation, a well-known feature in polymeric OLEDs, ${ }^{14,}{ }^{15}$ was not taken into account in small molecule OLEDs based on phosphorescent emitters for a long time. The reason is that the spherical appearance of the emitter molecules suggested an isotropic orientation particularly in amorphous matrices. However, recent studies have clearly shown that this is not generally the case. E.g. the common red phosphorescent emitter Iridium(III)bis(2-methyldibenzo[f,h]quinoxaline)(acetylacetonate) $\left(\operatorname{Ir}(\mathrm{MDQ})_{2}(\mathrm{acac})\right)$ doped with $8 \mathrm{wt} \%$ into a N,N'-bis(naphthalen-1-yl)-N,N'bis(phenyl)benzidine ( $\alpha$-NPD) matrix shows a predominantly horizontal orientation of the emitting dipoles. ${ }^{16}$ This means that the transition dipole moment is mainly lying in the plane parallel to the interfaces of the device. This feature strongly influences the efficiency analysis of OLEDs comprising this emitter. If its deviation from isotropy is not taken into account properly, the RQE of the emitting guest-host system is overestimated and hence the outcoupling factor is underestimated. ${ }^{17}$ In consequence, predictions of the power dissipation into different optical modes by optical simulations will be erroneous if an isotropic emitter orientation is assumed.

By means of optical simulations we discuss in which way oriented transition dipole moments of (phosphorescent) molecules affect the efficiency analysis. Especially the power dissipation into different optical channels and the determination of the RQE of the emitting guest-host system will be analyzed with respect to emitter orientation. Furthermore we present an outlook about possible efficiency enhancement by applying predominantly horizontally oriented emitters. 


\section{METHODOLOGY}

(a)

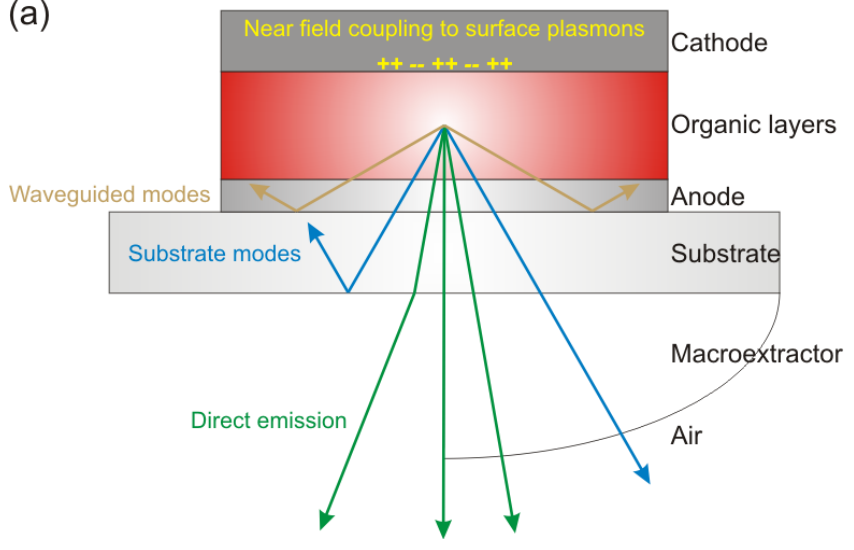

(b)

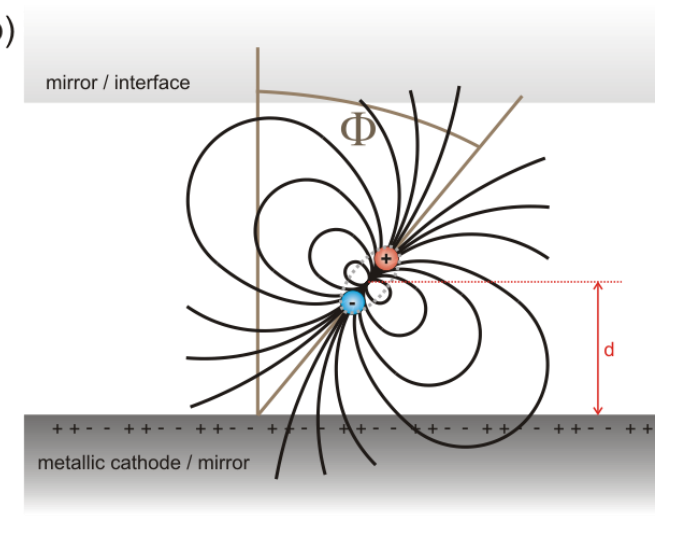

Figure 1: (a) Optical channels of light emission in an organic light emitting OLED with and without a macroscopic outcoupling structure. (b) Dipole in a microcavity-like structure. The radiative decay rate of the dipole is influenced by the Purcell effect.

Figure 1 illustrates the optical emission channels in an OLED and the influence of the mirror-like cathode and other interfaces on the dipole. Direct emission from an OLED is the part of the generated light that can pass the substrate/air interface and is determined by the angle of total internal reflection due to the mismatch of the refractive index of air and glass. Light propagating in the substrate above the critical angle of total internal reflection at the substrate/air interface is dissipated in the substrate modes and can be extracted with e.g. a glass hemisphere index-matched to the substrate. The remaining power is emitted into waveguided modes due to total reflection at the glass/organic interface or by near field coupling to surface plasmon polaritons at the metallic cathode. Figure 1b) shows a dipole in an OLEDlike microcavity structure.

The optical simulation used in this study is based on a classical dipole model from Barnes and Wasey, ${ }^{10,18}$ which solves the 3-dimensional Maxwell equations for an excited dipole emitter ensemble surrounded by multiple thin and planar layers with respect to electromagnetic boundary conditions. Considering important physical values as layer thicknesses, complex refractive indices, the position of the excited molecule with respect to the different interfaces (especially the emitter/metal distance), emitter orientation and the intrinsic radiative quantum efficiency (q) and using a transfer-matrix-formalism results in the power dissipation spectrum for the given dipole esemble. ${ }^{18,19}$

Thereby, the $x-y$-plane is oriented parallel and the z-axis perpendicularly to the surface of the OLED. Without loss of generality, the $\mathrm{x}-\mathrm{z}$-plane is chosen as the plane of observation, leading to the implication that the dipoles oriented along the $\mathrm{x}$-axis $\left(\mathrm{p}_{\mathrm{x}}, \mathrm{TM}_{\|}\right)$and along the $\mathrm{z}$-axis $\left(\mathrm{p}_{\mathrm{z}}, \mathrm{TM} \perp\right)$ can only emit p-polarized light, in contrast to the $\mathrm{y}$-dipole $\left(\mathrm{p}_{\mathrm{y}}, \mathrm{TE}_{\|}\right)$, which is only allowed to emit s-polarized light. From the electrodynamic radiation pattern of a dipole it follows that $\mathrm{p}_{\mathrm{x}}$ - and $\mathrm{p}_{\mathrm{y}}$-dipoles mainly radiate their energy perpendicularly to the surface and thus into direct emission or substrate modes, whereas $\mathrm{p}_{z}$-dipoles couple almost all energy into waveguided modes or to surface plasmons. ${ }^{10,20}$ Please note, that $\mathrm{p}_{\mathrm{x}}-$ and $\mathrm{p}_{\mathrm{y}}$-orientation are equivalent and the choice of two distinct $\mathrm{p}_{\mathrm{x}}$ - and $\mathrm{p}_{\mathrm{y}}$-dipoles originates from the selection of an observation plane only. Therefore, a superposition of $\mathrm{x}$ - and $\mathrm{y}$-dipoles has to be considered for horizontal emitter orientation.

The total radiative decay rate of an ensemble of excited molecules embedded in an infinite homogeneous medium $\left(\Gamma_{0}\right)$ can be expressed as the sum of the intrinsic radiative $\left(\Gamma_{\mathrm{r}}\right)$ and nonradiative $\left(\Gamma_{\mathrm{nr}}\right)$ decay rates 


$$
\Gamma_{0}=\Gamma_{r}+\Gamma_{n r}=\tau_{0}^{-1}
$$

and describes the power dissipation due to emission of light and non-radiative deactivation of excited states. The reciprocal value of the intrinsic total decay rate is the intrinsic lifetime $\tau_{0}$ of the excited molecule in such a medium. According to eq. (2) the intrinsic radiative quantum efficiency of the emitting system is given by:

$$
q=\frac{\Gamma_{r}}{\Gamma_{r}+\Gamma_{n r}} .
$$

If an emitter is embedded in a microcavity-like structure, such as an OLED, the radiative decay rate and thus the radiative quantum efficiency are modified by the so called Purcell effect. ${ }^{21-23}$ Due to different interference conditions for horizontal and vertical dipoles the Purcell factor $F$ must be calculated separately for each of the three orthogonal directions and is given by the following equation:

$$
F_{x, y, z}=\int_{\lambda_{1}}^{\lambda_{2}} S(\lambda) \int_{0}^{\infty} P_{x, y, z}\left(k_{\|}, \lambda\right) d k_{\|} d \lambda
$$

Here $S(\lambda)$ is the intrinsic emitter spectrum normalized to integral one. The integral over this spectrum is performed over the whole emitter spectrum. $\mathrm{P}_{\mathrm{x}, \mathrm{y}, \mathrm{z}}$ as a function of the in-plane wavevector $\mathrm{k}_{\|}$, represents the power dissipation of the dipoles in each direction and implicitly depends on the dipole position in the OLED. To account for orientation effects it is helpful to introduce an anisotropy factor $\Theta$ that is defined as the ratio between the amount of vertical dipoles and the total number of dipoles. According to this definition $\Theta$ becomes $1 / 3$ for isotropic emitter orientation, 1 for only vertical dipoles (dipoles in z-direction) and 0 for totally horizontal emitter orientation (all dipoles are lying in the $x$-y-plane)..${ }^{17}$ With this information the weighted Purcell factor can be calculated as

$$
F(\Theta)=\frac{1-\Theta}{2}\left(F_{x}+F_{y}\right)+\Theta \cdot F_{z} .
$$

Assuming non-radiative effects not to be altered due to the presence of the microcavity allows for modelling the changes in the radiative decay rate (eq. 6), ${ }^{24}$ in the effective radiative quantum efficiency (eq. 7) and in the relative changes of the lifetime of the excited molecules (eq. 8) ${ }^{17,23}$ :

$$
\begin{aligned}
& \tau(\theta)^{-1}=\Gamma(\theta)=F(\theta) \cdot \Gamma_{r}+\Gamma_{n r} \\
& q_{e f f}=\frac{F(\theta) \cdot \Gamma_{r}}{F(\theta) \cdot \Gamma_{r}+\Gamma_{n r}}=\frac{q \cdot F(\theta)}{(1-q)+q \cdot F(\theta)} \\
& \frac{\tau(\theta)}{\tau_{0}}=\frac{\Gamma_{0}}{\Gamma(\theta)}=[(1-q)+q \cdot F(\theta)]^{-1}
\end{aligned}
$$


Using this background a comprehensive efficiency analysis for arbitrary emitter orientation and for any OLED stack can be performed. In order to get information about e.g. the RQE of a given guest-host system a proper variation of the emitter-cathode distance is essential. ${ }^{25}$ By varying the thickness of the electron transporting layer it is possible to change the position of the emission layer with respect to the metallic mirror-like cathode. ${ }^{26}$ This in turn affects the power dissipation to the different optical modes and the effective RQE of the used guest-host system. By subsequently measuring the excited states lifetime changes and the EQE of the OLED stack with altered emitter-cathode distance and the prevailing emitter orientation by optical or electrical experiments it is possible to determine the intrinsic $\mathrm{RQE},{ }^{17}$ which is required for consistent simulation of the power dissipation into different optical channels.

\section{RESULTS AND DISCUSSION}

\subsection{Radiative quantum efficiency of a neat organic film}

Typically the RQE of a (new) emitting system is measured in a simple photoluminescence experiment of a neat film on glass in an integrating sphere. Thereby the number of emitted photons is divided by the number of absorbed photons giving an expression for the RQE. However, when assuming the presence of oriented emitters, this method can yield erroneous results due to two effects: First, the polarization of the pump beam might induce a preferred, oriented excitation. ${ }^{27}$ Second, the emission profile depends on the orientation distribution of the excited molecules. In order to demonstrate the possible over- or underestimations of the RQE of an emitting system, optical simulations for a neat film of a red guest-host system on a glass substrate have been performed for different emitter orientations. Figure 2 shows simulated power dissipation spectra as a function of the wavelength and the in-plane wavevector for the three orthogonal emitter orientations in a $10 \mathrm{~nm}$ thick $\alpha$-NPD layer doped with $8 \mathrm{wt} \% \operatorname{Ir}(\mathrm{MDQ})_{2}(\mathrm{acac})$. All results are normalized to the peak intensity of each dipole. Due to the thin organic layer and the absence of a metal, no guided modes or plasmons are present. It can clearly be seen, that the emission profile of the three dipole orientations is quite different. Whereas x- and y- dipoles emit a huge part of their power directly into air almost the whole power of the zdipole is dissipated into substrate modes. Due to missing outcoupling structures in such experiments these substrate modes could only be extracted at the edges of the glass substrate or due to scattering at imperfections of the film. However, a part of the radiation will always be reabsorbed and is lost. This clearly shows that the RQE obtained by an integrating sphere measurement is normally underestimated and this mistake will be largest, if only vertical dipole orientation is observed. The situation gets more complicated if thick organic layers are used. In this case radiation is additionally coupled into waveguide modes, especially for vertical emitter orientation. Most of the generated light that is captured in the organic layer cannot be extracted at the edges of the sample due to the huge mismatch of the refractive indices. This light stays in the organic layer and can there be reabsorbed or interact with excited molecules in an undesirable way. Furthermore, quenching effects at surfaces or interfaces,$^{28}$ especially for thin organic layers, should be considered in such experiments. With regard to cavity effects and eq. (7) the effective RQE of an emitting system is strongly influenced by the Purcell factor. For the organic film on a glass substrate a huge part of the radiation cannot be coupled out to air and is reflected at the organic/air and the glass/organic interface resulting in interference at the position of the emitting dipole inside the organic layer. The calculated Purcell factors are very different for each of the three directions. While the radiative rate of horizontal oriented emitters is not strongly influenced by the Purcell effect $\left(\mathrm{F}_{\|}=0.83\right)$, the radiative rate of vertical oriented emitters is almost completely suppressed $\left(\mathrm{F}_{\mathrm{z}}=0.15\right)$. This indicates that in such an experiment not the intrinsic lifetime and RQE of the guest-host system is measured but the effective RQE that is changed by the Purcell effect and this influence is strongest for vertical emitter orientation. If this is not taken into account, wrong radiative quantum efficiency will be determined. 

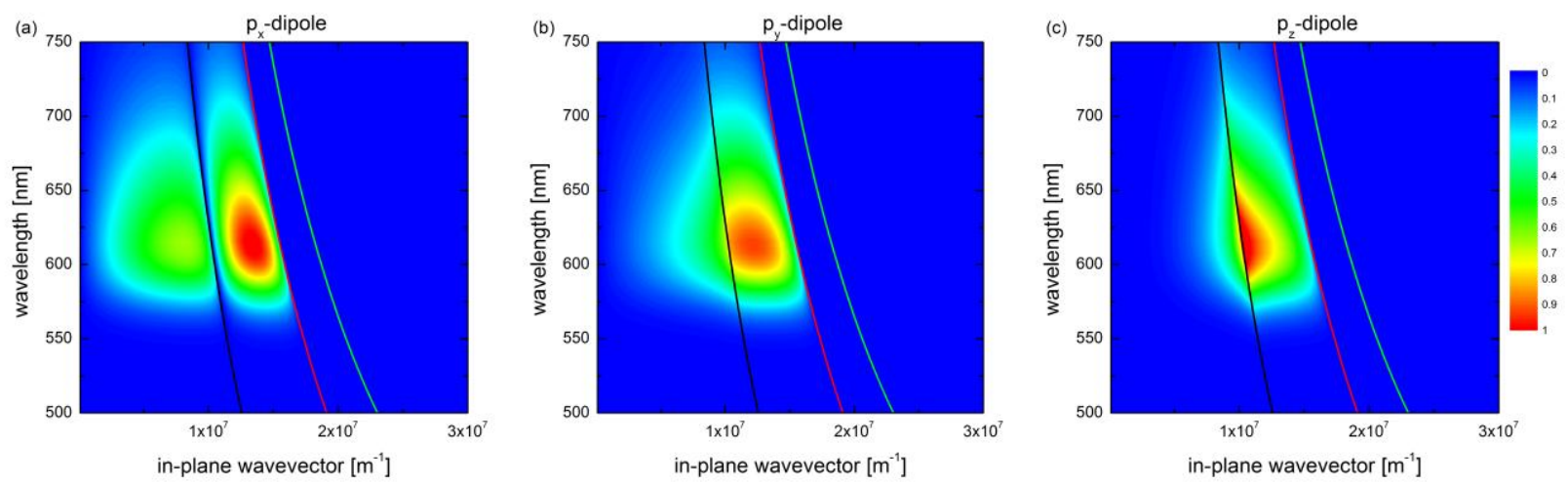

Figure 2: Separately simulated power dissipation spectra as a function of the wavelength and the in-plane wavevector for the three orthogonal emitter orientations in (a) $\mathrm{x}-$, (b) $\mathrm{y}$ - and (c) z-direction of $10 \mathrm{~nm}$ emission layer on a glass substrate normalized to each peak intensity. The black, red and green lines are the boundaries for direct emission, substrate modes and waveguided modes, respectively.

The reason for these low Purcell factors is the small layer thickness of the organic layer used in the simulation, resulting in destructive interference at the emitter position, especially for vertical oriented emitters. If a thicker film is used, the Purcell factors will only have little influence on the effective RQE, because they will be close to one, but other loss channels, such as waveguide modes in the thick organic will dominate the situation.

\subsection{Radiative lifetime changes in organic light-emitting diodes}

However, even if orientation and cavity effects were included in the RQE determination of a neat film on a glass substrate, the situation could change in the OLED stack due to different layer growth induced by the underlying materials. ${ }^{29,}{ }^{30}$ Ideally, the RQE is determined directly inside the OLED stack under investigation.

The state-of -the-art OLED stack and the intrinsic emitter spectrum are shown in Fig. 3. A hole injection and transport layer (HTL) is deposited onto an indium tin oxide (ITO) coated glass substrate followed by an electron blocking layer (EBL). Thereafter, an $\alpha$-NPD matrix was doped with $8 \mathrm{wt} \% \operatorname{Ir}(\mathrm{MDQ})_{2}(\mathrm{acac})$ (structure shown in Fig. 3) forming the thin emission layer (EML) of the device. In order to provide a high (close to unity) charge balance factor the emission layer is subsequently covered by an appropriate hole blocking (HBL) and an electron injection and transport layer (ETL) and finally contacted by a thick silver cathode. 

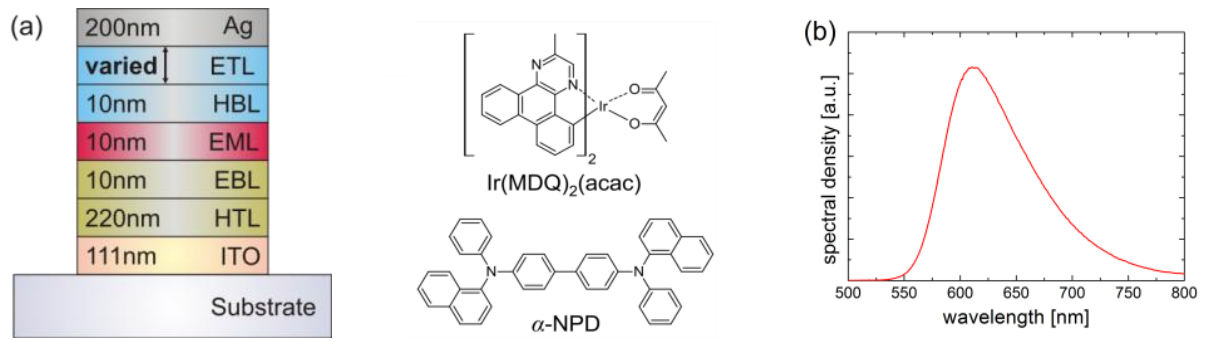

Figure 3: (a) Structure of the red phosphorescent bottom emitting OLED stack under investigation and the chemical structure of the host- $(\alpha-N P D)$ and the guest- $\left(\operatorname{Ir}\left(\mathrm{MDQ}_{2}(\mathrm{acac})\right)\right.$ molecule. The ETL layer thickness was varied between 20 and $400 \mathrm{~nm}$.

(b) Intrinsic red emitter spectrum with a peak wavelength at $610 \mathrm{~nm}$ used for all optical simulations

The thickness of the electron transport layer was varied to modify the interference conditions inside the OLED cavity. By changing the emitter-cathode distance, the effective RQE and the power dissipation into the different optical channels is strongly affected. Due to conductivity doping of the transport layers electrical changes through layer thickness variation can be minimized. Moreover, the charge balance factor and the singlet-triplet ratio remain constant for all transport layer thicknesses and both are assumed to be one.

$\operatorname{Ir}(\mathrm{MDQ})_{2}(\mathrm{acac})$ is a red phosphorescent emitter molecule with a predominantly horizontal emitter orientation in an $\alpha$ NPD matrix. ${ }^{16}$ The spectrum of the emitting system was measured under $0^{\circ}$ in a photoluminescence experiment using a thin emission layer evaporated on a glass substrate. The complex refractive indices of all layers as a function of wavelength have been determined in reflection-transmission measurements performed for each single layer separately with a neat film on glass. ${ }^{31}$

To achieve a comprehensive efficiency analysis including a quantification of the energy radiated into different optical channels, the next steps that have to be performed are: (1) determination of the emitter orientation inside the matrix material (ideally with the same surrounding of all organic and inorganic layers to ensure similar morphology dependent orientation effects ${ }^{29}, 30$ ), (2) determination of the (effective) radiative quantum efficiency of the guest-host system considering deviations from isotropy, and (3) finally computing the amount of power coupled to different optical cavity modes by means of optical simulations regarding the previous results.

The prevailing emitter orientation can be identified by means of two different experiments. The first method is a photoluminescence measurement of the emission spectrum as a function of the emission angle. ${ }^{12}$ Thereby it is necessary to quantify both, the s- and the p-polarized emission of the film. By analyzing the emission of $\mathrm{p}_{\mathrm{x}^{-}}$and $\mathrm{p}_{\mathrm{z}^{-}}$ dipoles and comparing this result with optical simulations performed for each dipole orientation it is possible to obtain the fraction of horizontal and vertical dipoles resulting in the anisotropy factor defined in section 2. In this experiment it is essential to fabricate the sample just as the device of interest but without the metallic cathode. In this case it is even possible to measure the emission of $\mathrm{p}_{z}$ dipoles under large angles as they cannot couple to surface plasmons due to the absence of a metal layer.

The second method is an electroluminescence experiment. ${ }^{15}$ In order to quantify the emitter orientation it is mandatory to fabricate the whole OLED choosing an appropriate ETL thickness. Due to different interference conditions for horizontally and vertically oriented dipoles there are emitter to cathode distances where direct emission of the $\mathrm{p}_{\mathrm{x}}-$ and $\mathrm{p}_{\mathrm{y}}$-dipoles is almost completely suppressed, while direct emission of the $\mathrm{p}_{\mathrm{z}}$-dipoles is enhanced. Analyzing the polarized emission patterns of such an OLED stack yields the vertical emitter contribution relative to that of the horizontal ones. 


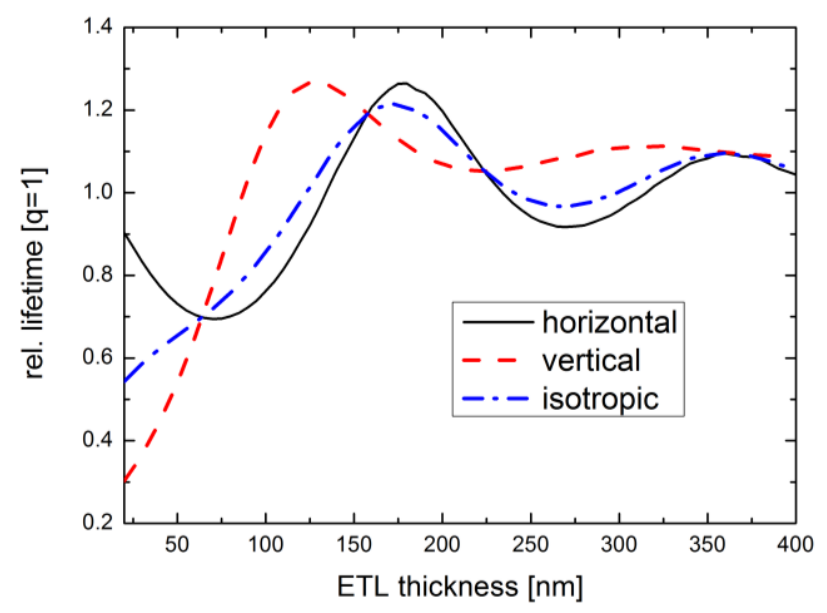

Figure 4: Separately simulated relative lifetime changes as a function of the electron transport layer thickness for horizontal, vertical and isotropic emitter orientation. The intrinsic radiative quantum efficiency was set to unity $(q=1)$.

Figure 4 shows the relative lifetime changes of three different dipole orientations for an intrinsic radiative quantum efficiency of $\mathrm{q}=1$ as a function of the ETL thickness. From eq. 8 it follows, that these relative lifetime changes are the reciprocal values of the Purcell factor, resulting from constructive and destructive interference conditions in the device under investigation. It can clearly be seen, that the local maxima for the relative lifetime changes are at different ETL thicknesses for vertical and horizontal emitter orientation. Additionally, the behavior for small emittercathode distances is completely different. Whereas horizontal dipoles only couple very weakly to surface plasmons, the vertical dipoles are dissipating almost all their energy to plasmons resulting in a very high Purcell factor and so in a strong reduction of their intrinsic lifetime. Please note, that this is only true for emitter/cathode distances larger than 15-20nm. For smaller ETL thicknesses the used dipole model is not valid and an energy transfer to lossy surface waves in the metal will occur. ${ }^{10,32}$

\subsection{Power distribution between optical modes in organic light-emitting diodes}

The diverse power dissipation of the different emitter orientations into the optical channels of an OLED is also reflected in the direct emission into air shown in Fig. 5. Here, the emission to air shows a local minimum for $\mathrm{p}_{\mathrm{x}}-$ and $\mathrm{p}_{\mathrm{y}}$-dipoles at ETL thicknesses, where $\mathrm{p}_{\mathrm{z}}$-dipoles exhibit their maximum. It should be noted, that emission to air is not vanishing for horizontal emitter orientation in the cavity minimum at about $160 \mathrm{~nm}$ due to the broad emission spectrum of the emitter used in the optical simulations (cf. Fig. 3). Furthermore these effects are exclusively related to variations of the outcoupling factor resulting from different coupling to optical modes inside the microcavity formed by the OLED. Please note, that the excited states lifetime is changed by the Purcell effect in this simulation, while the RQE of the emitting system remains constant for all ETL thicknesses due to the missing non-radiative decay rates (assumption: $\mathrm{q}=1$ ). 


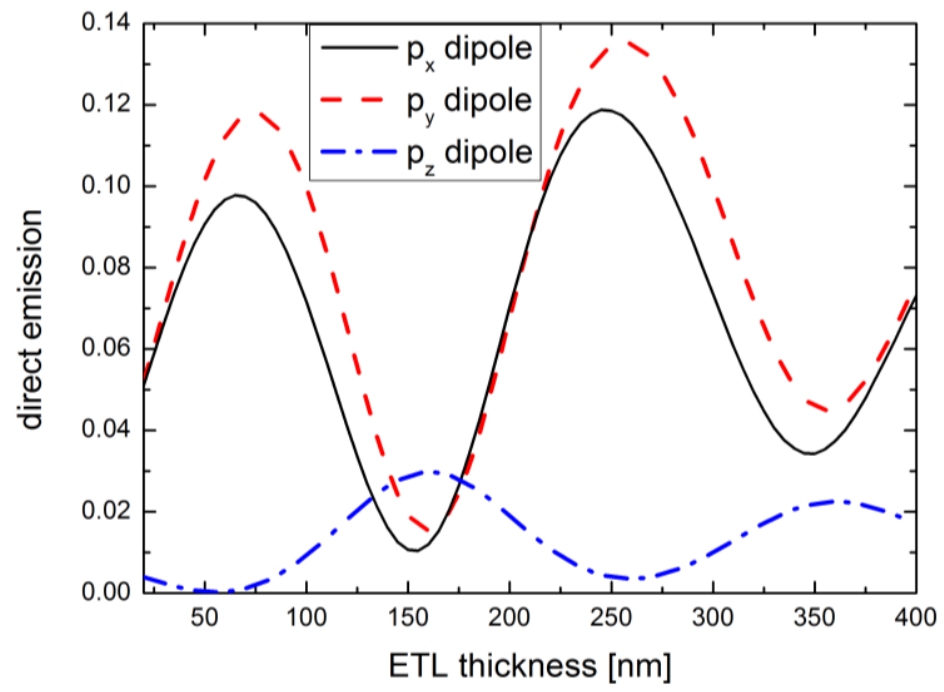

Figure 5: Separately simulated direct emission as a function of the electron transporting layer thickness for $p_{x^{-}}, p_{y^{-}}$and $p_{z}$-dipoles if isotropic emitter orientation is assumed. The intrinsic radiative quantum efficiency was set to unity $(\mathrm{q}=1)$.

For an ETL thickness of $160 \mathrm{~nm}$ the emission is mainly caused by vertical dipoles where $3 \%$ of their total power is dissipated into the direct emission mode, whereas horizontal emitters only dissipate $2.7 \%$ into this optical channel ( $1.5 \%$ and $1.2 \%$ for $\mathrm{p}_{\mathrm{y}^{-}}$and $\mathrm{p}_{\mathrm{x}}$-dipoles, respectively) if an isotropic emitter orientation is assumed. At this emittercathode distance a polarized measurement of the angular dependent spectra for an electrically driven OLED is very sensitive to emitter orientation, because the fraction of horizontal to vertical dipoles has a strong influence on the direct emission spectrum. ${ }^{15}$ This behavior becomes even more important if the total power dissipation of different dipole orientations is calculated.

Figure 6 demonstrates the power dissipation of isotropic, vertical and horizontal emitter orientation. Because the RQE of each dipole orientation was set to unity again the cavity effects only influence the power distribution between the optical channels. This changes if an emitting system with a RQE less than unity is used, as variations in the radiative emission rate due to the Purcell factor vary the relative effect of non-radiative losses. However, for the sake of simplicity this situation is not discussed in this work. For an isotropic emitter orientation a huge part of the dissipated power is coupled to surface plasmons for low emitter/cathode distances. For higher ETL thicknesses the coupling to plasmons vanishes and the emission to waveguided modes becomes the dominant effect. For ETL thicknesses above $200 \mathrm{~nm}$ almost $50 \%$ of the radiation is captured in waveguided modes and cannot be coupled out of the device in an easy way. However, the maximum in external quantum efficiency of this device is reached at an ETL thickness of 250 $\mathrm{nm}$ for direct emission, where almost $26 \%$ of the generated power can leave the device, and an EQE of $55 \%$ can be achieved, if substrate outcoupling structures are attached and an ETL thickness of $95 \mathrm{~nm}$ is used. 



Figure 6: Separately simulated power dissipation spectra as a function of the electron transporting layer thickness for (a) isotropic, (b) vertical and (c) horizontal dipole orientation. The intrinsic radiative quantum efficiency was set to unity $(q=1)$.

The situation changes if vertical emitter orientation is prevailing (see Fig. 6(b)). Here, the vertical dipoles are dissipating almost their whole energy to plasmons for ETL thicknesses below $100 \mathrm{~nm}$. This is due to the emission characteristics of an excited dipole that radiates most of its power perpendicular to its orientation and the exponential decay of the near-field coupling. ${ }^{33}$ Surprisingly, almost $53 \%$ of the total energy could be coupled out with macroscopic outcoupling structures that give access to the substrate modes for an ETL thickness of $190 \mathrm{~nm}$. In result, even a non-favorable vertical emitter orientation could achieve an EQE of up to 50\%, when using an emitting system with an intrinsic RQE of one ( $\mathrm{q}=1)$. As soon as the ETL thickness exceeds $190 \mathrm{~nm}$ the biggest amount of energy of the vertical oriented dipoles is coupled to p-polarized waveguided modes. However, the choice of the emitter/cathode distance must be considered in a completely different way than for an isotropic emitter orientation. Nevertheless, vertical emitter orientation should be avoided due to the strong angular dependent emission of the excited substrate modes.

In contrast, the favorable horizontal emitter orientation shows a completely different behavior. Figure 6 (c) depicts the enormous potential of this orientation. Even for small emitter/cathode distances weak coupling to surface plasmons appears due to the lack of vertical dipoles. Furthermore, the power distribution between waveguided and substrate modes differs significantly from the isotropic case. A comparison of isotropic and horizontal emitter orientation 
emphasizes the huge potential of increasing the external quantum efficiency of OLEDs. If only direct emission is of interest an optimized device with an isotropic emitter orientation can yield 26\% EQE for an ETL thickness of $250 \mathrm{~nm}$, while the horizontal emitter distribution could reach an EQE of $37 \%$ for the same ETL thickness. This is an impressive increase of $42 \%$ (rel.) of the isotropic value. Moreover, if macroscopic outcoupling structures, such as glass hemispheres index-matched to the substrate, are used and all substrate modes are coupled out, the situation becomes even more remarkable. In the isotropic case an optimized device can achieve a maximum EQE of 55\% for an ETL thickness of $95 \mathrm{~nm}$. Indeed the horizontal emitter distribution could easily beat this value. For an ETL thickness of $73 \mathrm{~nm}$, an external quantum efficiency of $74 \%$ could be achieved. This is a significant efficiency boost by almost $35 \%$ (rel.). It should be pointed out that this EQE enhancement is possible without any additional costs in fabrication, if an emitter is used that shows a totally horizontal emitter distribution in the surrounding matrix.

With this information it is now possible to address the issue of determining the radiative quantum efficiency for a given guest-host system in a proper way considering possible deviations from isotropy.

\subsection{Determination of the radiative quantum efficiency in complex OLED structures}

To investigate the RQE of an emitting system in an OLED stack a subsequent variation of the ETL thickness as described above is needed. According to sec. 2 the effective radiative quantum efficiency is influenced by the microcavity formed by the metallic cathode and the substrate of an OLED. Due to different coupling to the optical modes when changing the emitter-cathode distance, the radiative rate and so the effective RQE is varied. Thus it is possible to extract the intrinsic RQE of the emitting guest-host system (and the effective RQE for every single OLED stack) either by measuring excited states lifetime changes as a function of the ETL thickness or by quantifying the external quantum efficiency for the electrically driven OLEDs with different emitter-cathode distances. For these two experiments it is essential to know the prevailing emitter orientation to perform a consistent RQE analysis. As described in eq. (5)-(8), simulations have been performed to analyze the relative changes of the excited state lifetime for the OLED stack shown in Fig. 3 (a).

To illustrate the importance of including possible deviations from isotropy for RQE determination by means of excited state lifetime changes, Fig. 7 shows simulations for different emitter orientations and different intrinsic radiative quantum efficiencies as a function of the ETL thickness.

It is obvious that the determination of the RQE of the emitting system will be erroneous, if orientation effects are not taken into account for these lifetime changes. Figure 7(a) shows the simulated relative lifetime changes of the given guest-host system for different intrinsic radiative quantum efficiencies as a function of the ETL thickness for an isotropic emitter orientation. Subsequently measuring the excited states lifetime for different emitter/cathode distances, deriving the ratio of the local maximum to the local minimum of the relative lifetime changes and comparing this value with the simulation yields the intrinsic RQE. ${ }^{11}$ The situation becomes more complicated if the used guest-host system shows a non-isotropic emitter orientation. Figure 7(b) shows the simulated lifetime behavior for the given OLED stack as a function of the ETL thickness for different orientations and intrinsic radiative quantum efficiencies. This clearly demonstrates, that comparable lifetime changes can be explained by both an isotropic emitter orientation with an intrinsic RQE of $90 \%$ or horizontal emitter orientation and an intrinsic RQE of $65 \%$. RQE determination by analyzing the ratio of the local extremes, as mentioned before, will not yield an unambiguous result, because both assumptions (isotropic simulation with a RQE of $90 \%$ and horizontal orientation with a RQE of $65 \%$ ) will yield similar lifetime data. The only larger discrepancies of the simulations occur at very small ETL thicknesses. Here, the emission rate is boosted by strong near-field coupling to surface plasmons in the isotropic case resulting in a high Purcell factor and a short excited state lifetime. If horizontal emitter orientation is assumed, coupling to surface plasmons for small emitter/cathode distances is almost negligible due to the angular dependent emission of an electrical dipole. In an experiment where these emitter-cathode distances are not considered, deviations to the simulation would not be apparent, leading to an efficiency analysis with a wrong emitter orientation and a wrong intrinsic RQE. This shows the importance of using a large variation of the emitter/cathode distance, especially for small ETL thicknesses to provide a consistent efficiency analysis including emitter orientation and cavity effects. 

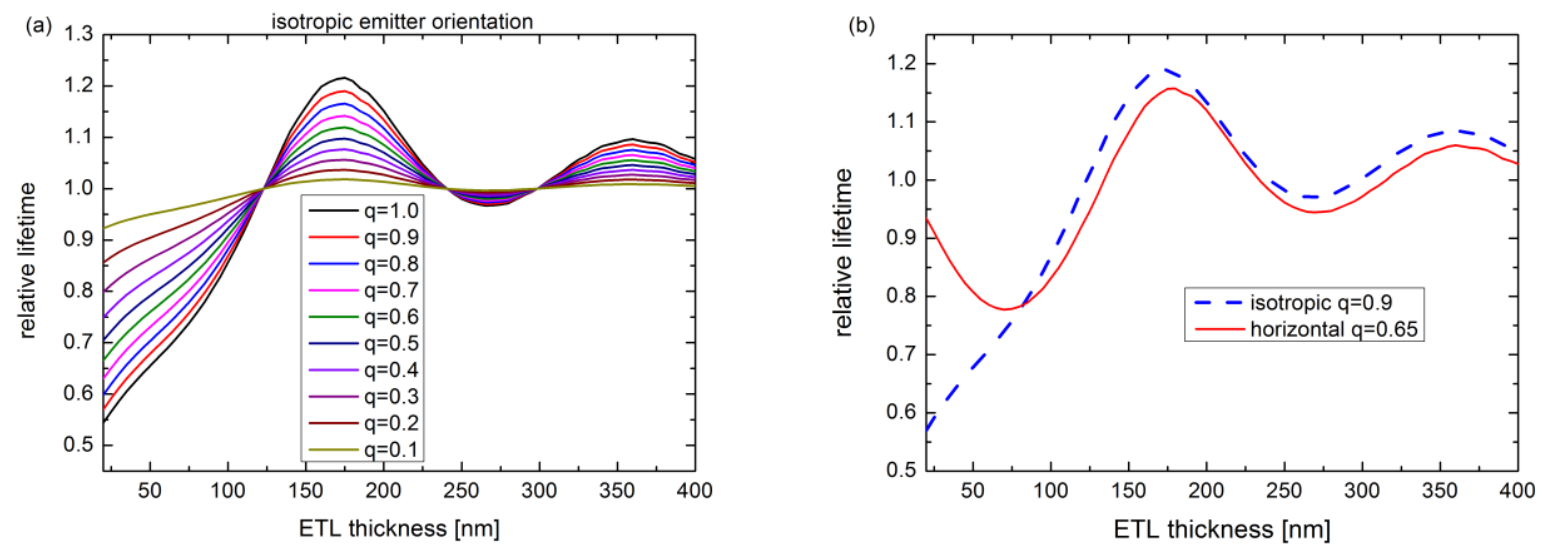

Figure 7: Simulated relative lifetime changes for a red phosphorescent OLED as a function of the ETL thickness. (a) Lifetime changes for an isotropic emitter orientations for different intrinsic RQEs. (b) Comparison of relative lifetime changes for the OLED under investigation for isotropic emitter orientation with an intrinsic RQE of $90 \%$ and for a horizontal emitter orientation (anisotropy factor $\Theta=0$ ) with an intrinsic radiative quantum efficiency of $65 \%$.

The discrepancies become even more obvious, if the RQE is additionally determined by electrically driven EQE measurements in an integrating sphere. Please note, that an estimation of the EQE from $0^{\circ}$ intensity measurements would be completely wrong, because oriented emitters do not show a Lambertian emission profile and thus this method is not applicable at all. Figure 8 shows simulations of the external quantum efficiency for the OLED under investigation for different emitter orientations and intrinsic RQEs as a function of the electron transporting layer thickness. It can be seen that both simulations (isotropic and horizontal emitter orientation with an intrinsic RQE of $90 \%$ and $65 \%$, respectively) match very well for the direct emission. However, the analysis becomes inconsistent, if an additional macoextractor is used that can couple out all substrate modes of the OLED. Then the different orientations show a huge deviation. Due to the different coupling to optical channels and the unequal influence of the Purcell effect on the effective RQEs, the substrate modes show a remarkably different behavior for the isotropic case than for the horizontal emitter orientation especially for ETL thicknesses between $100 \mathrm{~nm}$ and $250 \mathrm{~nm}$. In this range, horizontal emitters yield a rather low EQE as compared to the isotropic case for the assumed RQEs. Thus, only measuring the direct emission will lead to wrong estimations of the RQE and so other factors of the EQE could be under- or overestimated. This shows the importance of using all possible information to ensure a comprehensive efficiency analysis especially for oriented emitting systems. The effect of orientation is remarkable. Although the RQE of the horizontally oriented emitters is only $65 \%$, higher maximum EQE values for slightly different ETL thicknesses than for the isotropic orientation with a RQE of $90 \%$ are possible. This indicates the huge potential of horizontal oriented emitters to boost OLED efficiencies. 


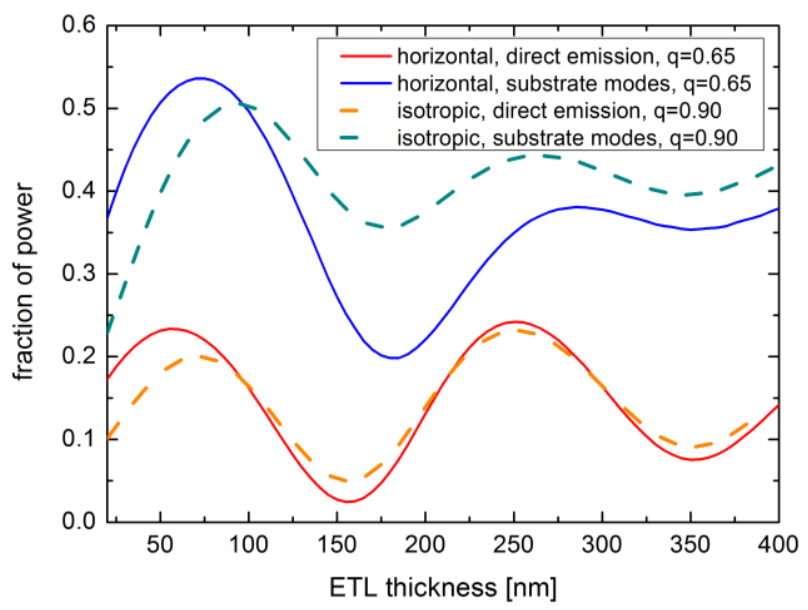

Figure 8: Simulated external quantum efficiencies (with and without a macroscopic outcoupling structure) as a function of ETL thickness for different emitter orientations and RQEs.

Furthermore, these simulations clearly show the difficulties of providing a consistent efficiency analysis for oriented emitting systems used in OLEDs. Measured lifetime and EQE data interpreted in a wrong way due to missing data points for the right ETL thicknesses could result in a considerable overestimation of the RQE of emitting systems leading to a wrong optimization of the OLED by means of thickness adjustment of the different organic layers.

In order to emphasize the importance of the discussed simulations, the basic findings were confirmed for the OLED given in figure 2. Figure 9 shows the determination of the radiative quantum efficiency for the OLED under investigation for different emitter orientations via lifetime and $\mathrm{EQE}$ measurements. Whereas an isotropic emitter orientation with a RQE of $80 \%$ cannot explain all measured data points, especially for the EQE characteristics with an outcoupling structure, simulations for an orientation of the emitters with an anisotropy factor $\Theta=0.65 / 2.65=0.24$ show a nearly perfect agreement. This clearly demonstrates that considering possible deviations from isotropy for the emitter orientation in common state-of-the-art OLEDs is essential to provide a consistent efficiency analysis leading to a proper optimization for highly efficient OLEDs.
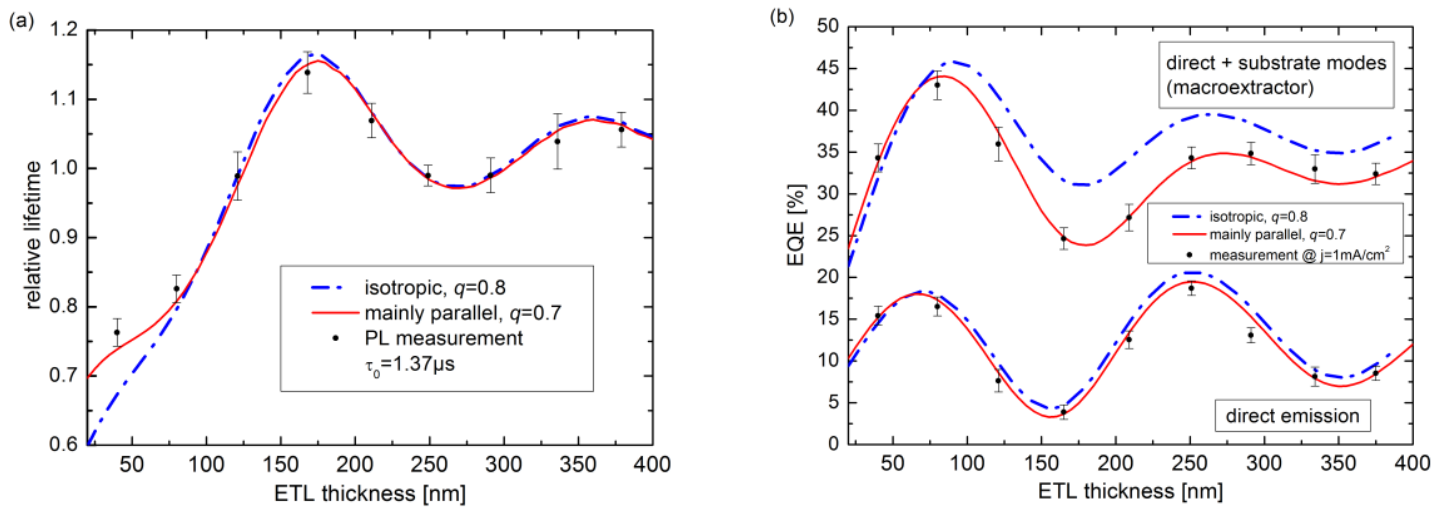

Figure 9: Determination of the intrinsic RQE for the given OLED stack with the prevailing emitter orientation of $\Theta=0.65 / 2.65$. (a) via the excited states lifetime changes and (b) via EQE measurements. In order to show the problems due to emitter orientation, isotropic simulations are added to the graphs. (Experimental data points are taken from Ref. 17.) 


\section{CONCLUSION}

In conclusion, the effect of emitter orientation on experimental efficiency analysis of organic light-emitting diodes based on optical simulations has been discussed, emphasizing possible error sources that prevent a consistent efficiency analysis of state-of-the-art OLED stacks. Neglecting possible deviations from isotropy yields wrong estimations for the radiative quantum efficiency and for other limiting efficiency factors, like the singlet/triplet ratio (especially for fluorescent emitters), and the outcoupling efficiency. According to our results, two different approaches can be followed in order to obtain a reliable light source analysis. First, emitter orientation can be measured directly using optical ${ }^{12}$ or electrical ${ }^{34}$ excitation combined with proper analysis of the polarized emission patterns. Second, comparing relative lifetime changes of different devices should include small emitter cathode distances (Fig. 7) to allow for estimating the orientation distribution. In result, efficiency measurements with and without substrate macroextractors should be used as cross check for the consistency of assumptions and results (Fig. 9). If all measurements are performed properly, a consistent analysis of the power radiated into the different optical channels can be achieved. For the system under study a theoretical limit of $74 \%$ external quantum efficiency for substrate emission was calculated when utilizing a completely horizontally oriented emitting system. A macroscopic outcoupling structure or outcoupling foils attached to the substrate could couple at least a large part of this power into air, not requiring expensive and complicated attachments like high-index substrates or Bragg-gratings implemented into the OLED structure.

\section{ACKNOWLEDGMENT}

The authors want to thank the German Federal Ministry of Education and Research (BMBF) for financial support under the Contract No. FKZ 13N10474 (TOPAS 2012). 


\section{References}

[1] Tang C. W. and VanSlyke S. A., "Organic electroluminescent diodes,” Appl. Phys. Lett. 51, 913-915 (1987).

[2] Y.-S. Tyan, "Organic light-emitting-diode lighting overview," J. Photon. Energy 1,011009 (2011).

[3] Gather M. C., Köhnen A., and Meerholz K., "White Organic Light-Emitting Diodes," Adv. Mater. 23, 233248 (2011).

[4] Tsutsui T., Aminaka E., Lin C. P., and Kim D.-U., "Extended molecular design concept of molecular materials for electroluminescence: sublimed-dye films, molecularly doped polymers and polymers with chromophores," Phil. Trans. R. Soc. Lond. A 355, 801-813 (1997).

[5] Baldo M. A., O’Brien D. F., You Y., Shoustikov A., Sibley S., Thompson M. E., and Forrest S. R., "Highly efficient phosphorescent emission from organic electroluminescent devices," Nature 395, 151-154 (1998).

[6] Nowy S., Krummacher B. C., Frischeisen J., Reinke N. A., and Brütting W., "Light Extraction and optical loss mechanisms in organic light-emitting diodes: influence of the emitter quantum efficiency," J. Appl. Phys. 104 (12), 123109 (2008).

[7] Greenham N. C., Friend R. H., and Bradley D. D. C., "Angular dependence of the emission from a conjugated polymer light-emitting diode: Implications for efficiency calculations," Adv. Mater. 6, 491-494 (1994).

[8] Nowy S., Frischeisen J., and Brütting W., "Simulation based optimization of light-outcoupling in organic light-emitting diodes," Proc. SPIE 7415, 74151C (2009).

[9] Smith L. H., Wasey J. A. E., Samuel I. D. W., and Barnes W. L., "Light out-coupling efficiencies of organic light-emitting diode structures and the effect of photoluminescence quantum yield," Adv. Funct. Mater. 15, 1839-1844 (2005).

[10] Barnes W. L., "Fluorescence near interfaces: The role of photonic mode density," J. Mod. Opt. 45, 661-699 (1998).

[11] Setz D. S., Schmidt T. D., Flämmich M., Nowy S., Frischeisen J., Krummacher B. C., Dobbertin T., Heuser K., Michaelis D., Danz N., Brütting W., and Winnacker A., "Comprehensive efficiency analysis of organic light-emitting devices," J. Photon. Energy. 1, 011006 (2011).

[12] Frischeisen J., Yokoyama D., Adachi C., and Brütting W., "Determination of molecular dipole orientation in doped fluorescent organic thin films by photoluminescence measurements," Appl. Phys. Lett. 96, 073302 (2010).

[13] Frischeisen J., Yokoyama D., Endo A., Adachi C., and Brütting W., "Increased light outcoupling efficiency in dye-doped small molecule organic light-emitting diodes with horizontally oriented emitters," Org. Electron. 12, 809-817 (2011).

[14] Wasey J. A. E., Safonov A., Samuel I. D. W., and Barnes W. L., "Effects of dipole orientation and birefringence on the optical emission from thin films," Opt. Commun. 183, 109-121, (2000).

[15] Flämmich M., Gather M. C., Danz N., Michaelis D., Bräuer A. H., Meerholz K., and Tünnermann A., "Orientation of emissive dipoles in OLEDs: Quantitative in situ analysis," Org. Electron. 11, 1039-1046 (2010).

[16] Flämmich M., Frischeisen J., Setz D. S., Michaelis D., Krummacher B.C., Schmidt T. D., Brütting W., and Danz N., "Oriented phosphorescent emitters boost OLED efficiency," Org. Electron. 12, 1663-1668 (2011).

[17] Schmidt T. D., Setz D. S., Flämmich M., Frischeisen J., Michaelis D., Krummacher B. C., Danz N., and Brütting W., "Evidence for non-isotropic emitter orientation in a red phosphorescent organic light-emitting diode and its implications for determining the emitter's radiative quantum efficiency," Appl. Phys. Lett. 99, 163302 (2011).

[18] Wasey J. A. E. and Barnes W. L., "Efficiency of spontaneous emission from planar microcavities," J. Mod. Opt. 47, 725-741 (2000).

[19] Chance R. R., Prock A., and Silbey R., "Molecular fluorescence and energy transfer near interfaces," Adv. Chem. Phys. 37, 165 (1978).

[20] Gaertner G. and Greiner H., "Light extraction from OLEDs with (high) index matched glass substrates," Proc. SPIE 6999, 69992T (2008). 
[21] Neyts K. A., "Simulation of light emission from thin-film microcavities," J. Opt. Soc. Am. A 15, 962-971 (1998).

[22] Chen X.-W., Choy W. C. H., Liang C. J., Wai P. K. A., and He S., "Modifications of the exciton lifetime and internal quantum efficiency for organic light-emitting devices with a weak/strong microcavity," Appl. Phys. Lett. 91, 221112 (2007).

[23] Wasey J. A. E., Safonov A., Samuel I. D. W., and Barnes W. L., "Efficiency of radiative emission from thin films of a light-emitting conjugated polymer," Phys. Rev. B 64, 205201 (2001).

[24] Mladenovski S., Reineke S., and Neyts K., "Measurement and simulation of exciton decay times in organic light-emitting devices with different layer structures," Opt. Lett. 34 (9), 13751377 (2009).

[25] Nowy S., Reinke N. A., Frischeisen J., and Brütting W., "Light extraction and optical loss mechanisms in organic light-emitting diodes," Proc. SPIE 6999, 69992V (2008).

[26] Flämmich M., Gather M. C., Danz N., Michaelis D., and Meerholz K., "In situ measurement of the internal luminescence quantum efficiency in organic light-emitting diodes," Appl. Phys. Lett. 95, 263306 (2009).

[27] Danz N., Heber J., Bräuer A., and Kowarschik R., "Fluorescence lifetimes of molecular dye ensembles near interfaces, “ Phys. Rev. A 66, 063809 (2002).

[28] Beierlein T.A., Ruhstaller B., Gundlach D. J., Riel H., Karg S., Rost C., and Riess W., "Investigation of internal processes in organic light-emitting devices using thin sensing layers," Synth. Met. 138, 213-221 (2003).

[29] Wagner J., Gruber M., Hinderhofer A., Wilke A., Bröker B., Frisch J., Amsalem P., Vollmer A., Opitz A., Koch N., Schreiber F., and Brütting W., "High fill factor and open circuit voltage in organic photovoltaic cells with diindenoperylene as donor material," Adv. Func. Mater. 20, 4295-4303 (2010)

[30] Dürr A. C., Koch N., Kelsch M., Rühm A., Ghijsen J., Johnson R. L., Pireaux J.-J., Schwartz J., Schreiber F., Dosch H., and Kahn A., "Interplay between morphology, structure, and electronic poperties at diindenoperylene-gold interfaces," Phys. Rev. B 68, 115428 (2003).

[31] M. Flämmich, N. Danz, D. Michaelis, A. Bräuer, M. C. Gather, J. H.-W. M Kremer, and K. Meerholz, "Dispersion-model-free determination of optical constants: application to materials for organic thin film devices," Appl. Opt. 48 (8), 1507-1513 (2009).

[32] Celebi, K., Heidel, T. D., and Baldo, M. A., "Simplified calculation of dipole energy transport in a multilayer stack using dyadic Green's functions," Opt. Express, 15 (4), 1762-1772 (2007).

[33] Pitarke J. M., Silkin V. M., Chulkov E. V., and Echenique P. M., "Theory of surface plasmons and surfaceplasmon polaritons," Rep. Prog. Phys. 70, 1-87 (2007).

[34] Flämmich M., Michaelis D., Danz N., "Accessing OLED emitter properties by radiation pattern analysis,“ Org. Electron. 12, 83-91 (2011). 